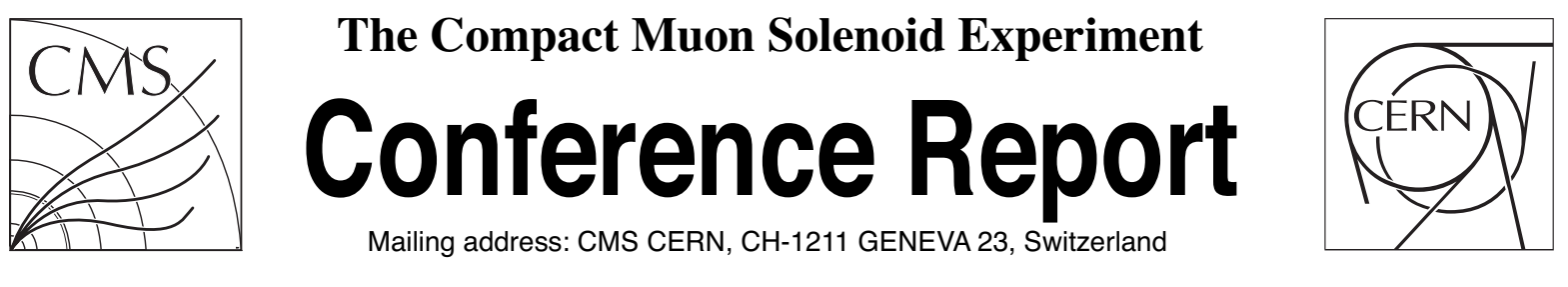

23 November 2017 (v3, 14 December 2017)

\title{
The CT-PPS project detector hardware and operational experience
}

Fabio Ravera, on behalf of the CMS and TOTEM Collaborations

\begin{abstract}
The CMS-TOTEM Precision Proton Spectrometer allows extending the LHC physics program by measuring protons in the very forward regions of CMS. Tracking and timing detectors have been installed along the beam pipe at $\sim 210 \mathrm{~m}$ from the CMS interaction point on both sides of the LHC tunnel. The tracking system consists of a station of silicon strip detectors and one of silicon pixel detectors on each side. The latter is composed of six planes of 3D silicon pixel sensors bump-bonded to the PSI46dig ROC developed for the CMS Phase I Pixel Tracker upgrade. A track resolution of $\sim 10 \mu \mathrm{m}$ is obtained. The future goal is to replace the present strip stations with pixel ones in order to ensure better multi-track reconstruction. Each timing station is made of three planes of diamond detectors and one plane equipped with an Ultra-Fast Silicon Detector (UFSD). A timing resolution of a few tens of picoseconds can be achieved with the present detector; a large R\&D effort is ongoing to reach the $10 \mathrm{ps}$ target resolution. This contribution describes the hardware characteristics and the present status of the CT-PPS project. The operational experience during the 2017 data taking is also presented.
\end{abstract}




\title{
The CT-PPS project: detector hardware and operational experience
}

\author{
F. Ravera*, on behalf of the CMS and TOTEM Collaborations \\ University of Genova, INFN Genova \\ E-mail: fabio.raveraecern.ch
}

The CMS-TOTEM Precision Proton Spectrometer allows extending the LHC physics program by measuring protons in the very forward regions of CMS. Tracking and timing detectors have been installed along the beam pipe at $\sim 210 \mathrm{~m}$ from the CMS interaction point on both sides of the LHC tunnel. The tracking system consists of a station of silicon strip detectors and one of silicon pixel detectors on each side. The latter is composed of six planes of 3D silicon pixel sensors bumpbonded to the PSI46dig ROC developed for the CMS Phase I Pixel Tracker upgrade. A track resolution of $\sim 10 \mu \mathrm{m}$ is obtained. The future goal is to replace the present strip stations with pixel ones in order to ensure better multi-track reconstruction. Each timing station is made of three planes of diamond detectors and one plane equipped with an Ultra-Fast Silicon Detector (UFSD). A timing resolution of a few tens of picoseconds can be achieved with the present detector; a large R\&D effort is ongoing to reach the $10 \mathrm{ps}$ target resolution. This contribution describes the hardware characteristics and the present status of the CT-PPS project. The operational experience during the 2017 data taking is also presented.

The 26th International Workshop on Vertex Detectors

10-15 September, 2017

Las Caldas, Asturias, Spain

${ }^{*}$ Speaker. 


\section{Introduction}

The aim of the CMS-TOTEM Precision Proton Spectrometer (CT-PPS) project [1] is the measurement of Central Exclusive Production (CEP) in the standard, high-luminosity fills at the Large Hadron Collider (LHC). CEP in proton-proton collisions, namely the process $p p \rightarrow p X p$, can be driven by a photon-photon interaction or double Pomeron exchange. The advantage of measuring this reaction is that, by reconstructing the two leading protons and specifically their fractional momentum loss $(\xi)$ and four-momentum transfer squared $(t)$, the full event is measured and strong constraints can be imposed on the object X reconstructed by the central CMS detector.

CT-PPS already acquired data in 2016 with an "accelerated program" and with $\sim 10$ of the $\sim 15 \mathrm{fb}^{-1}$ collected, the first measurement of semi-exclusive dimuon production at the LHC energies has been published [2]. From the reconstructed proton it is possible to constrain the dimuon kinematics, allowing a strong suppression of the backgrounds, mainly due to Drell-Yan dimuons and pile-up protons. Thanks to that, with the only 12 events observed a significance of $4.3 \sigma$ has been reached. This analysis, together with proving the effectiveness of the CT-PPS detector, shows also the discover potential of adding a near-beam proton spectrometer to the experiments at LHC.

\section{The CT-PPS detector}

The CT-PPS detector consists of tracking and timing detectors installed between 200 and $220 \mathrm{~m}$ from the Interaction Point (IP) on both sides of CMS. Each arm of LHC is equipped with one timing and two tracking stations. During 2016, CT-PPS took data using as tracking devices the silicon strips developed for the TOTEM experiment. In 2017 one of the tracking stations in each side has been equipped with 3D silicon pixel detectors in order to improve the radiation hardness and the multiple track reconstruction efficiency. The reason to gradually upgrade the detector was driven by the possible data-taking efficiency loss due to the commissioning time required by the newly installed stations. The full transition to a pixel tracking detector is foreseen for the 2018 data taking. The timing detector was installed at the end of the 2016 data taking and was originally made of diamond planes. In 2017 one of the planes has been replaced by an Ultra-Fast Silicon Detector (UFSD), making CT-PPS the first high energy physics application for this technology.

In order to insert them close to the beam (between 1.5 and $2.5 \mathrm{~mm}$ depending on the station), the detectors are installed in movable devices called Roman pots (RP). The tracking detectors are installed within rectangular RPs with the same design as those from TOTEM and upgraded with a radio-frequency shield in order to reduce the impact on the machine impedance. A $150 \mu \mathrm{m}$ thick steel window allows to reduce the distance between the detector and the beam, improving the acceptance at lower $\xi$. The tracking station is composed of three RPs: one horizontal, which is the only one inserted in high luminosity fills, and two vertical, above and below the beam pipe, which overlap the horizontal RP. The vertical RPs are only used during alignment runs (see sec. 3).

For the timing detectors, a new cylindrical RP design was developed for CT-PPS providing both a larger space to host the detectors and also reducing the impedance impact on the LHC beam-line. In this design the thin window is $300 \mu \mathrm{m}$ thick.

Both types of Roman pots are inserted in high luminosity fills at 12 beam sigmas plus a safety margin of $0.3 \mathrm{~mm}$. The detectors within the pot are placed in a secondary vacuum in order to 
reduce the stress on the thin window, which on the other side faces the high vacuum of the LHC beam-pipe.

\subsection{The tracking detector}

By measuring the proton tracks at the RP positions it is possible to determine the proton kinematics at the IP by means of the so-called beam optics, which provides a parametrization of the LHC magnetic lattice between the IP and the RPs.

Because of the small distance from the LHC beam at which the detectors are operating, good radiation hardness is required. The simulations indicate that for $100 \mathrm{fb}^{-1}$ a maximum flux of the order of $\sim 5 \times 10^{15}$ proton $/ \mathrm{cm}^{2}$ is expected, which corresponds to fluences between 1 and $3 \times 10^{15} \mathrm{neq} / \mathrm{cm}^{2}$. Besides the radiation hardness, another requirement is the reduction of the insensitive area at the sensor edge facing the beam, in order to increase the acceptance to lower masses of the centrally produced state.

Tracking sensors are operated at $\sim-20^{\circ} \mathrm{C}$ in order to mitigate the increase of the leakage current due to the irradiation. Therefore, to avoid condensation, the pressure in the tracking RPs is kept below 20 mbar.

The baseline design of the CT-PPS tracking detector foresees to use 3D pixel sensors that provide an intrinsic radiation hardness and the possibility of effectively implementing slim edges. In the following sections the design and the production of the 3D modules for CT-PPS is described along with the strip detectors used for the first years of data taking.

\subsubsection{TOTEM strip detector}

The TOTEM strips were developed with a micro-strip edge-less technology on a $300 \mu \mathrm{m}$ silicon substrate [3]. Current terminating rings around the active area of the sensor are implemented in order to collect the large current generated in the highly damaged sensor edge, and reduce the insensitive area down to $50 \mu \mathrm{m}$. The strips, oriented at $\pm 45^{\circ}$ with respect to the edge closer to the beam, have a pitch of $66 \mu \mathrm{m}$ and are binary read-out with the VFAT2 ReadOut Chip (ROC) [4].

The tracking station is equipped with 5 units each made of two strip sensors with opposite strip orientation. A track resolution of $10 \mu \mathrm{m}$ is reached in both $\mathrm{x}$ and $\mathrm{y}$ directions. Apart from the limitations in reconstructing events with multiple tracks, the lifetime of these sensors is limited to $\sim 5 \times 10^{14}$ proton $/ \mathrm{cm}^{2}$ and hence the detectors need to be replaced during the data taking period.

The back-end electronics and the data acquisition (DAQ) software are the same as those of the TOTEM experiment and were integrated into the CMS DAQ system at the beginning of 2016.

\subsubsection{D pixel detector}

The basic concept of the 3D sensors [5] is that, instead of implementing the electrodes on the two wafer surfaces like it is done for the standard planar technology, they are etched in a column perpendicularly to the surface towards the silicon bulk. This design allows to decouple the detector thickness, which is proportional to the charge deposited by a crossing particle, and the inter-electrode distance. This provides several advantages: lower depletion voltage, faster charge collection and high charge collection efficiency after irradiation.

Several beam tests and irradiation campaigns have been carried out on the 3D sensors from different vendors - CNM (Barcelona, Spain), FBK (Trento, Italy) and SINTEF (Oslo, Norway) - 
and the suitability of this technology for CT-PPS has been confirmed. The order was eventually placed with CNM.

The CNM sensors for CT-PPS have been implemented in a double-sided non-passing-through technology, meaning that $n^{+}$and $p^{+}$electrodes are etched from opposite surfaces of the wafer and they stop before reaching the other surface (see Fig. 1a). The sensors have been built on a $230 \mu \mathrm{m}$ thick wafer and the columns are $200 \mu \mathrm{m}$ deep. A $200 \mu \mathrm{m}$ slim edge is implemented with $p$-type column fences surrounding the sensitive area. By increasing the bias voltage, the inefficient edge can be reduced down to $50 \mu \mathrm{m}[6]$.

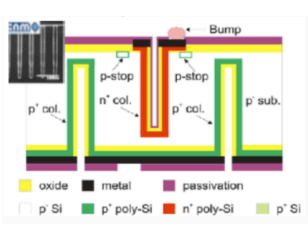

(a) CNM 3D cross section.

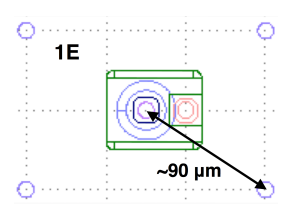

(b) 1E pixel layout.

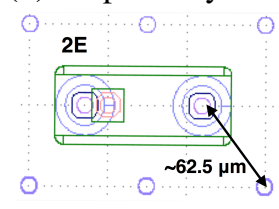

(c) 2E pixel layout.

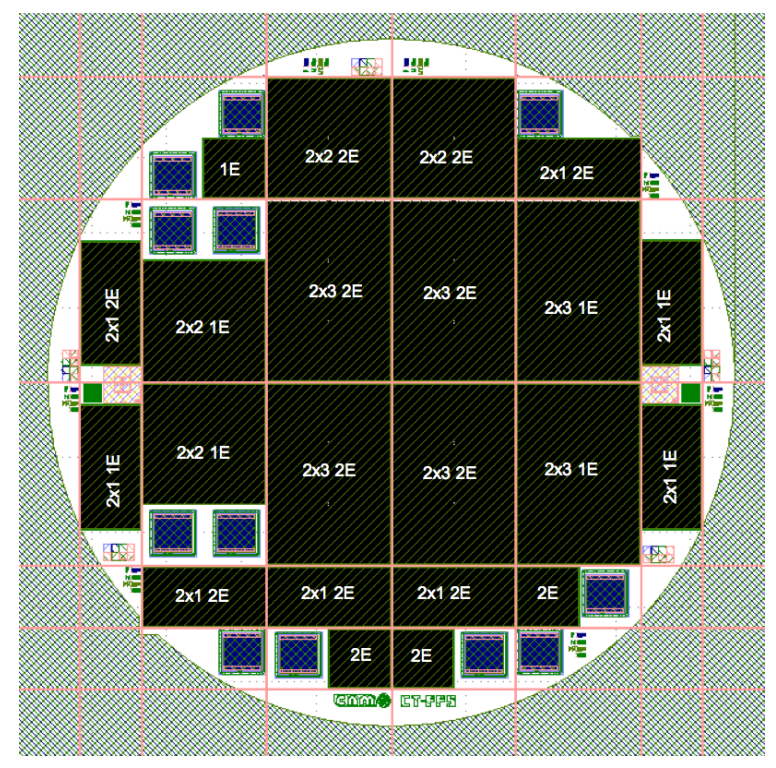

(d) Wafer layout for the CT-PPS 3D sensors produced at CNM.

Figure 1: Characteristics of the CNM 3D sensor production for CT-PPS.

The production of the 3D sensors has been divided into three batches of 12 wafers whose layout is shown in Fig. 1d. The sensors are designed to be read out by the PSI46dig ReadOut Chip (ROC), the same used for the CMS Phase I pixel detector [7], which is made of a $52 \times 80$ matrix of pixels with size $150 \times 100 \mu \mathrm{m}^{2}$. The baseline design for CT-PPS is a $2 \mathrm{E}$ pixel configuration (two readout columns for every pixel as shown in Fig. 1c) and $2 \times 3$ sensors, which means readout by 6 ROCs. However, also $1 \mathrm{E}$ (one readout column for every pixel as shown in Fig. 1b) and $2 \times 2$ sensors are included in the wafer design as a backup solution. In the layout also single-ROC sensors are present and used for validating the production on beam [6].

The qualification of the sensors is done on wafer before the dicing by measuring the IV characteristic at room temperature by means of a temporary metal deposition that shorts all the pixels of the module [8]. Sensors are requested to have a breakdown voltage higher than $35 \mathrm{~V}$ and if the requirement is fulfilled, they are classified in class $\mathrm{A}, \mathrm{B}$ and $\mathrm{C}$ if the leakage current per ROC is $I<2 \mu \mathrm{A}, 2 \mu \mathrm{A}<I<10 \mu \mathrm{A}$ and $I>10 \mu \mathrm{A}$, respectively. Class $\mathrm{C}$ sensors are rejected.

The first batch was completed in December 2015 with in general good quality sensors, but low class A yield. Therefore, in order to produce the stations installed in 2017, sensors with both dimensions $(2 \times 3$ and $2 \times 2)$ and both pixel layouts $(1 \mathrm{E}$ and $2 \mathrm{E})$ were used allowing to obtain 
46 modules, which guarantee the assembly of the needed detectors. The second batch, completed in May 2016, showed sensors with very low breakdown voltage and the whole production was rejected. The problem was attributed by CNM to the $p$-stop implantation carried out by an external company. Finally, the third batch completed in June 2017 had sensors with large leakage current, which would classify all the modules as class C. After discussing with the PSI46dig designers it was decided to relax the class B current limit well above the ROC specifications and accept sensors with a leakage current up to $400 \mu \mathrm{A}$ per ROC, allowing to accept further $\sim 50$ modules.

After bump-bonding at IZM, Berlin, Germany, the modules were tested in the Torino and Genova laboratories, and the final modules have been selected for the installation. Each tracking station is composed of 6 planes tilted by $18.4^{\circ}$ in order to increase the charge sharing and therefore improve the resolution. Each plane is made of a 3D module glued on a Thermal Pyrolithic Graphite (TPG) layer that provides the thermal contact to the cooling circuit, which was adapted from the TOTEM one. The module is wire bonded to a flex circuit that allows the connection to the so-called RPix portcard. This board, developed by INFN Genova, is derived from TOTEM strip detector portcard with the components of the CMS Phase I pixel upgrade, and interfaces the modules to the back-end electronics, which is identical to the one of the CMS pixel detector.

Two pixel detector packages were mounted at CERN and tested in the same conditions of cooling and secondary vacuum as those present along the LHC beam line. The two RP units were installed along the LHC beam-pipe in March 2017 during the extended year-end technical stop (EYETS). At the same time the DAQ software was developed and integrated in the CMS central DAQ. By means of the ROC internal calibration circuit, the detector was fully calibrated and optimized for data taking. The read-out threshold was set to $\sim 2000 \mathrm{e}^{-}$and the spread between pixels was measured to be $\sim 135 \mathrm{e}^{-}$. The percentage of bad channels, defined as those with efficiency lower than $90 \%$ in measuring a charge of $\sim 5000 \mathrm{e}^{-}$, is less that $0.05 \%$.

\subsection{The timing detector}

CT-PPS has to operate in high luminosity at LHC with up to 50 pile-up interactions per bunch crossing. It is therefore crucial to associate the protons measured with CT-PPS with the vertex reconstructed in CMS, especially in the cases in which the central detector does not reconstruct all the final-state products (neutrinos or beyond standard model objects) and the kinematic cuts constraining the central system and the proton are less effective. In order to achieve this, a " $\mathrm{z}$ by-timing" method is used, based on the measurement of the difference of arrival times of the two protons in opposite directions. In particular, with a $10 \mathrm{ps}$ timing resolution, a vertex position resolution of $\sigma_{z} \sim 2 \mathrm{~mm}$ can be obtained, which would allow to strongly suppress the pile-upinduced backgrounds.

Due to the higher voltage at which the diamond detectors are operated $(\sim 500 \mathrm{~V})$, the pressure in the timing RPs is set between 100 and 110 mbar in order to avoid discharging. For this reason the temperature of the cooling circuit is set to $15^{\circ} \mathrm{C}$.

Both technologies are connected to a discrete-component amplifier that shapes the signal to be fed into the NINO amplifier [9], whose output is then digitized by the High-Precision Time-toDigital Converter (HPTDC) [10], which provides the measurement of the leading and trailing edge allowing for the time-walk correction. The coded signal is then handled by an FPGA close to the 
detector that encodes the data and sends them to the back-end electronics, which is adapted from the TOTEM one.

In order to reach the target resolution, the detector must be synchronized with a clock with jitter of the order of few ps. The present detector has two different systems that can provide the precise clock: the Universal Picosecond Timing System based on an optical network and an RFfeedback with low-loss coaxial cable. Both systems showed comparable figures of merit.

Each timing station is equipped with four planes of timing detectors. While in 2016 all the planes were equipped with diamond detectors, in 2017 each station consists of three diamond and one Ultra-Fast Silicon Detector (UFSD) planes.

\subsubsection{Diamond detector}

Each of the three diamond planes is made of four $4 \times 4 \mathrm{~mm}^{2}$ single crystals produced by chemical vapor deposition. The diamond sensors were grown to a thickness of $500 \mu \mathrm{m}$ by Element Six Ltd., Ascot, UK, and the metallization of the pads was done at Applied Diamond, Inc., Wilmington, USA, and PRISM, the Princeton University laboratory. Each of the four crystals of the plane has a different segmentation along the $\mathrm{x}$ direction in order to equalize the channel occupancy and hence smaller pads are present closer to the beam. Crystals with the same segmentation are glued with different orientation and position between planes to improve the x-resolution to $\sim 150 \mu \mathrm{m}$ and allow a rough track reconstruction. The orientation of the diamond pads is visible in the plots of Fig.3

The timing resolution measured on beam is $\sim 80$ ps per plane [11] and since the technology is intrinsically radiation hard, the sensors are expected to withstand the whole proton flux CT-PPS is expected to be exposed to.

\subsubsection{UFSD}

The UFSD sensors installed in CT-PPS were produced at CNM; they have an active thickness of $50 \mu \mathrm{m}$. The sensor pads have been adapted to couple with the diamond plane readout and hence eight $0.5 \times 6 \mathrm{~mm}^{2}$ and four $1 \times 3 \mathrm{~mm}^{2}$ pads are presently connected (see Fig.3).

This kind of technology showed on beam a timing resolution of $\sim 35$ ps [12]. The radiation hardness for the UFSD is still an issue and they are expected to withstand up to $\sim 10^{14}$ proton/ $\mathrm{cm}^{2}$. Significant R\&D on this aspect is ongoing.

\section{Operational experience and status of the data taking}

An integral part of the CT-PPS data taking are the so-called alignment runs. This special run has multiple goals: RP alignment with respect to the collimators, relative alignment between the RPs, alignment with respect to the beam and optics determination.

The procedure starts by closing the collimators upstream of the detector to few beam sigmas (between 5 and 8.5) and afterwards slowly approaching one by one the RPs to the beam. When a spike is observed in the Beam Loss Monitors (BLM) downstream of the detector the pot has reached the same position as the collimators in terms of beam sigmas. At this point the collimators are opened and the data taking starts. 
The relative alignment between the RPs is determined by the tracks passing in the overlap regions of the vertical and horizontal pots, while the alignment with respect to the beam is performed by analysing the hit distributions from protons in elastic scattering events in the vertical detectors and in diffractive events in the horizontal detectors. Finally, the tuning of the optics, which is fundamental for the proton kinematic reconstruction, is obtained by analysing the hit distribution shape in the horizontal tracking detectors. While the global alignment for the 2017 has already been determined, the optics parameter tuning requires a more refined analysis that is currently in progress.

In Fig. 2 the track $x$ and y coordinates are shown for the two tracking stations on each side of CMS. Although the coordinates are expressed in arbitrary systems of reference for each Roman pot, the halo visible on the vertical pots (top and bottom) corresponds to the edge of the hit distribution in the horizontal RP, confirming the overlap of the detectors, a fundamental requirement for the alignment studies. It is also worth noticing that this is the first time the CT-PPS pixel detector took data as a subdetector of CMS.

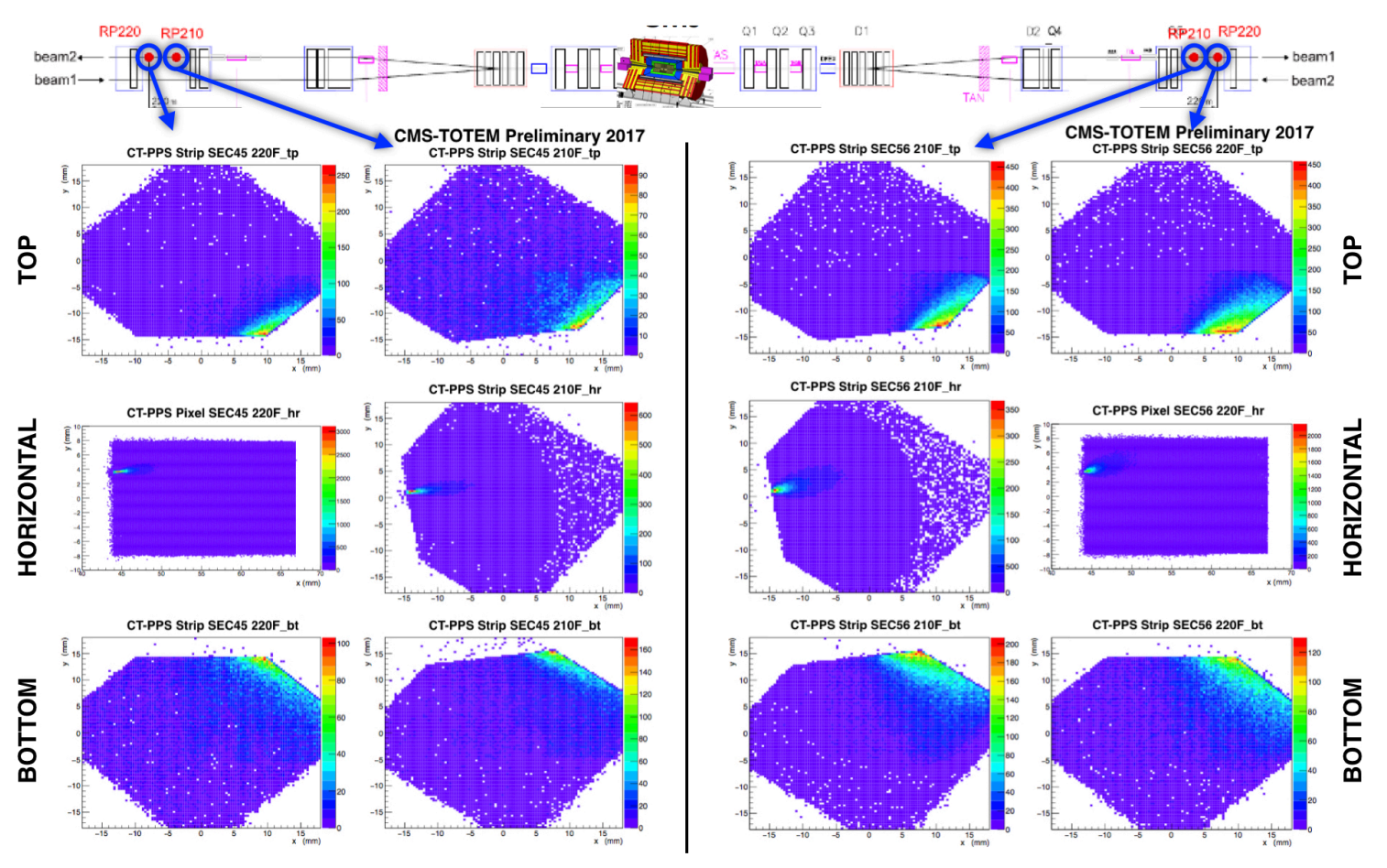

Figure 2: Track position distribution on the tracking CT-PPS detectors during the alignment run.

Even if the timing detector is still in a commissioning phase, it has been possible to verify the consistency of the data collected so far. In order to do that, Fig. 3 shows for each of the planes installed on one of the two arms the hit maps measured in the strip detector by requiring a coincidence in the timing plane considered (similar plots are obtained for the opposite side). Despite the low statistics, one can recognize the expected hit distribution as shown in the tracking detectors (Fig. 2), which indicates that the sensors are centered on the vertical beam position. The different entry colors correspond to different fired channels of the plane allowing also to verify that the detectors are correctly mapped. 

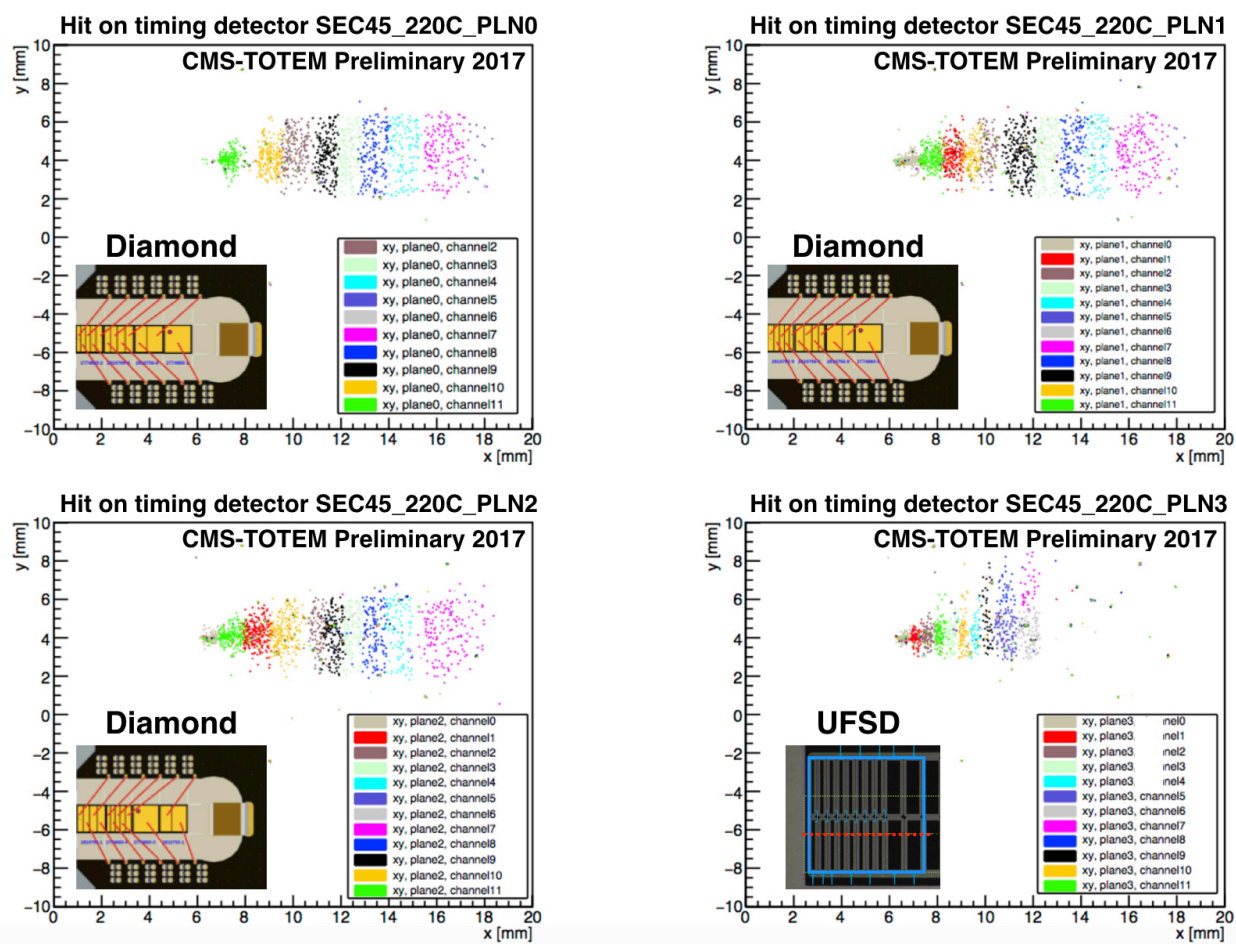

Figure 3: Hit maps as measured in the strips are plotted requiring a coincidence with timing detectors. Different colors correspond to different channels fired on the plane. As a comparison, the corresponding pad layout is shown.

As already mentioned, CT-PPS took data also in 2016 and collected data corresponding to an integrated luminosity of $\sim 15 \mathrm{fb}^{-1}$. In 2017, after the alignment run, the Roman Pots have been inserted during the LHC intensity ramp-up and certified for insertion up to the maximum 2017 luminosity with 2556 bunches. After this step, they have been inserted in all the high luminosity fills of the year and recorded $\sim 40 \mathrm{fb}^{-1}$ (non official number). The detector ran stably during the whole data taking with minimum impact on the CMS data acquisition.

\section{Conclusions}

The current CT-PPS detector has been installed in April 2017 and is composed of four different detector technologies: silicon strips, silicon 3D pixels, scCVD diamonds and UFSDs. All the detectors have been successfully integrated in the CMS DAQ since the first fill of 2017. The 3D pixel tracker, which constitutes the first application of this technology in CMS, has been commissioned. The timing detector system, which includes the first application of UFSD in a high energy physics experiment, has shown to meet expectations. A full analysis of its performance is currently ongoing. So far CT-PPS recorded $\sim 55 \mathrm{fb}^{-1}$ and $10 \mathrm{fb}^{-1}$ of them have been analyzed to produce its first physics result. CT-PPS is foreseen to collect data in 2018 with a full 3D pixel tracker that will further improve the performance in order to reach the $100 \mathrm{fb}^{-1}$ design target. 


\section{Acknowledgment}

Special thanks go to the CT-PPS group for the great commitment that allowed the success of the installation and data taking. We would also like to express our gratitude to the 26th International Workshop on Vertex Detectors for inviting us to present the CT-PPS project.

\section{References}

[1] The CMS and TOTEM Collaborations, CMS-TOTEM Precision Proton Spectrometer, CERN-LHCC-2014-021 (2014)

[2] The CMS and TOTEM Collaborations, Evidence for proton-tagged, central semi-exclusive production of high-mass muon pairs at $13 \mathrm{TeV}$ with the CMS-TOTEM Precision Proton Spectrometer, CMS-PAS-PPS-17-001, TOTEM-NOTE-2017-003 (2017)

[3] The TOTEM Collaboration, Final size planar edgeless silicon detectors for the TOTEM experiment, Nucl. Instrum. Meth. A563 (2006), 41

[4] P.Aspell et al., VFAT2: A front-end system on chip providing fast trigger information, digitized data storage and formatting for charge readout of multi-channel silicon and gas particle detectors, Electronics for particle physics. Proceedings, Topical Workshop, TWEPP-07, Prague, Czech Republic, September 3-7 (2007), 67

[5] S. Parker et al., 3-D: A New architecture for solid state radiation detectors, Nucl. Instrum. Meth. A 395 (1997), 328

[6] F. Ravera, The CT-PPS tracking system with 3D pixel detectors, JINST 11 (2016) C11027

[7] The CMS Collaboration, CMS Technical Design Report for the Pixel Detector Upgrade, CERN-LHCC-2012-016 (2012)

[8] C. Da Via et al., 3D Silicon Sensors: Design, Large Area Production and Quality Assurance for the ATLAS IBL Pixel Detector Upgrade, Nucl. Instrum. Meth. A694 (2012) 321

[9] F. Anghinolfi et al., NINO: an ultra-fast and low-power front-end amplifier/discriminator ASIC designed for the multigap resistive plate chamber, Nucl. Instrum. Meth. A533 (2004) 183

[10] M. Mota and J. Christiansen, A high-resolution time interpolator based on a delay locked loop and an RC delay line, IEEE JSSC 34 (1999) 1360

[11] G Antchev et al., Diamond detectors for the TOTEM timing upgrade, JINST 12 (2017) P03007

[12] N. Cartiglia et al., Beam test results of a 16 ps timing system based on ultra-fast silicon detectors, Nucl. Instrum. Meth. A850 (2017) 83 